\title{
COMPLIANCE CONTROL OF A LEGGED ROBOT BASED ON IMPROVED ADAPTIVE CONTROL: METHOD AND EXPERIMENTS
}

\author{
Yaguang $\mathrm{Zhu}^{*}$ and Bo Jin**
}

\begin{abstract}
For the purpose of impact reduction and stable walking of a hexapod robot under different environments, a control strategy based on the improved adaptive control algorithm is proposed. According to the model of robot structure and impedance control, a fuzzy control scheme is utilized for parameters adjustment of adaptive control. With a real-time error regulator, a fast response of system can be achieved. Comparative analysis and a series of experiments for improved compliance control method are proposed in different circumstances and presence of disturbs. The results show that expected force can be tracked quickly in complex environments, as well as smaller contact shocking and body height fluctuations are obtained, which makes foot force regulating speed of the legs be more balance and decreases instability risk of the whole system. With this characteristic, foot slipping in soft terrains can be avoided. This means the proposed strategy has great benefit for the adaptability and robustness of the hexapod walking robot in complex environment.
\end{abstract}

\section{Key Words}

Adaptive control, walking robot, foot tip force, adaptability, compliance control

\section{Introduction}

In recent years, multi-legged walking robot has become a hot research topic for its good obstacle avoidance capability and strong adaptability to walk on the complex surface. In the future, broad application prospects of multi-legged walking robot can be theoretically guaranteed by its good environmental adaptability and motion flexibility, since its flexible motion capability comes from a lot of freedom of the leg. Generally, the foot position points can be obtained on the foot trajectory planning and gait equations. But the robot may be unstable if there are errors in position

${ }^{*}$ Key Laboratory of Road Construction Technology and Equipment of MOE, Chang'an University, China; e-mail: zhuyaguang@chd.edu.cn

** State Key Laboratory of Fluid Power \& Mechatronic Systems, Zhejiang University, China; e-mail: bjin@zju.edu.cn

Recommended by Prof. Enver Tatlicioglu

(DOI: 10.2316/Journal.206.2016.5.206-4536) planning or foot forces imbalance on soft environments [1]. Since the coordinate system of the robot is attached to the body, the coordinate system is not fixed regards with ground. This leads to inaccurate measurement of the surface sinking. Thus, the compliance control becomes a key point for smooth walking and tasks, and it is also the premise of fast walking, trotting, and even running [2].

Two approaches can be used for implementation of impedance control: position-based impedance control and force/torque-based impedance control [3]. In the first approach, Silva et al. [4] establishes a control strategy to make the robot more stable and more robust in different environments. A PD controller is used to deal with the feedback. Rönnau et al. [5] introduces a model-based position/force controller, in which a computed torque control approach is used to decrease the nonlinear disturbances. Many other researchers such as Oku et al. [6] and Pavone et al. [7] utilize these strategies. The second approach is also broadly used in mobile robot control. Yin et al. [8] provides an impedance control to enhance the adaptability of walking for the robot with few sensors. Galvez et al. [9] presents a design for feet and ankles with an integrated sensor system to minimize the slippage risk by force optimization schemes. Actually, many other methods have been tried [10]-[12] to solve the control problems such as coupling of force and position, degree of freedom multiplicity, and uncertain posture in mobile robotics. However, current walking robots have only poor ability of leg compliance control to complete complex tasks in an unknown environment.

In this article, a novel control method is presented for improvement of the indirect adaptive method [13]. A fuzzy controller makes foot force regulating speed of the legs be more balanced by adjusting parameters, which will decrease instability risk of the whole system. Experiments on a two-leg test rig are conducted and they proved that the expected force can be tracked accurately by using the proposed method, which have benefit in force impact elimination. Foot slipping can be avoided in soft terrains for the fast converge of force tracking, which is also helpful for improvement of the robustness and adaptability. The advantage of adaptive fuzzy is that a faster convergence of speed will be maintained in whatever environment, and 


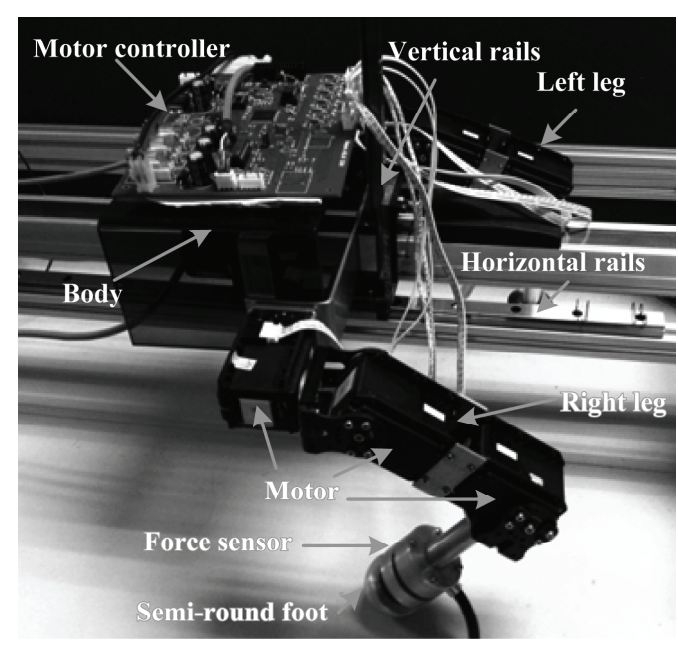

Figure 1. Test rig for compliance control.

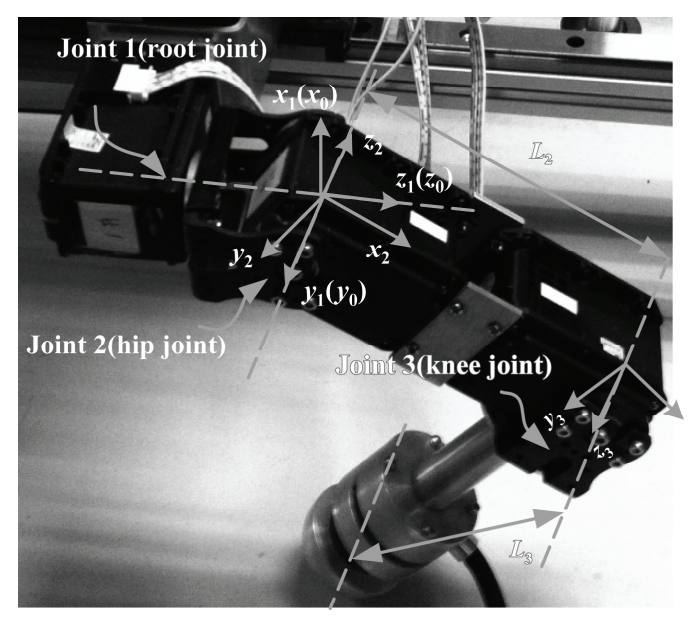

Figure 2. Reference frames of the leg.

hence in view of whole body locomotion, the contribution for stable walking on complex terrain will be remarkable.

\section{Mechanical Structure of Walking Robot}

In this article, the main body of robot is supported with legs in a mammal-like configuration since mammal legs require less joint torques to support the body [14]. The characteristic of low energy consumption and large load is more suitable for process of transport work. The test rig is shown in Fig. 1. Each leg consists of three links which are connected by a hip joint and a knee joint. The leg mechanism is attached to the body via a root joint. Here, a semi-round foot with certain radius is used for its significant advantages in adapting to complex terrain [15]. Since the test rig is designed for the compliance control, horizontal and vertical rails with corresponding displacement sensors, a five-dimensional force sensor, and LPC2368-based motor controller are equipped.

According to the Denavit-Hartenberg convention, the kinematic model of the robot is established in Fig. 2 [14]. The leg structure of the hexapod robot is optimized by
Table 1

$D-H$ Parameters

\begin{tabular}{|l|r|c|c|c|}
\hline Linkage $j$ & $\alpha_{i} /{ }^{\circ}$ & $d_{i} / \mathrm{m}$ & $a_{i} / \mathrm{m}$ & $\theta_{i} /{ }^{\circ}$ \\
\hline 1 & 90 & 0 & 0 & $-35-45$ \\
\hline 2 & 0 & 0 & $L_{2}=0.103$ & $74-168$ \\
\hline 3 & 0 & 0 & $L_{3}=0.141$ & $49-148$ \\
\hline
\end{tabular}
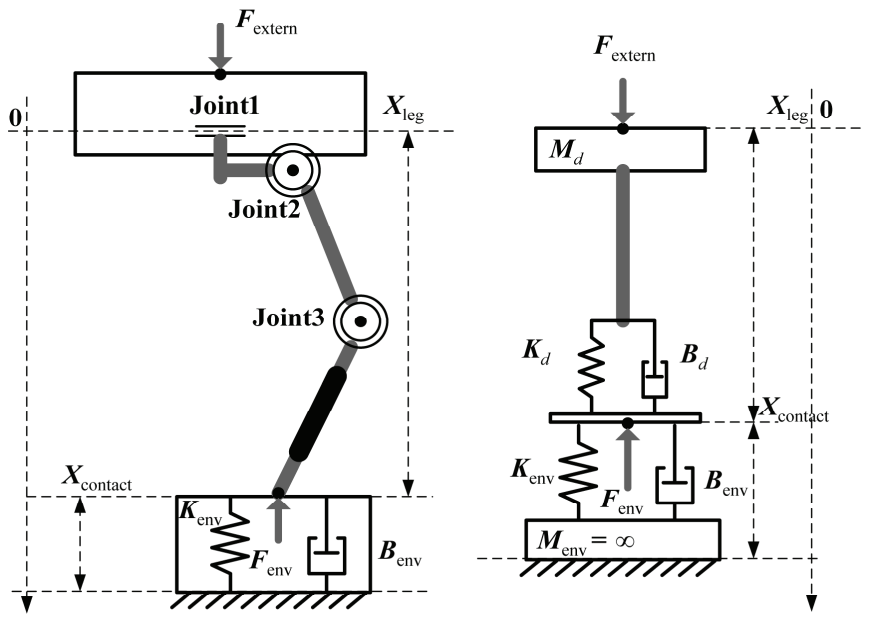

Figure 3. Equivalent mechanical scheme.

workspace and energy consumption [16]. The parameters of Denavit-Hartenberg are shown in Table 1.

\section{Position-based Impedance Control}

The impedance control algorithm establishes a desired user-specified dynamical relationship referred to as the "target impedance" between the contact force $F$ and the foot tip position $X$. Normally, the relationship between $F$ and $X$ can be supposed as a mass-spring-damper system, for a linear second-order equation is chosen. The target impedance can be shown in formulation:

$$
\mathbf{M}_{\mathbf{d}}\left(\ddot{\mathbf{X}}-\ddot{\mathbf{X}}_{\mathbf{r}}\right)+\mathbf{B}_{\mathbf{d}}\left(\dot{\mathbf{X}}-\dot{\mathbf{X}}_{\mathbf{r}}\right)+\mathbf{K}_{\mathbf{d}}\left(\mathbf{X}-\mathbf{X}_{\mathbf{r}}\right)=-\mathbf{F}
$$

in which $\mathbf{M}_{\mathbf{d}}, \mathbf{B}_{\mathbf{d}}$, and $\mathbf{K}_{\mathbf{d}}$ are the $n \times n$ constant diagonal mass, damping, and the target impedance stiffness matrices. $\mathbf{F}$ is the foot tip contact force. $\mathbf{X}$ and $\mathbf{X}_{\mathbf{r}}$ stand for the actual and reference trajectories, respectively. The equivalent mechanical scheme is shown in Fig. 3. There is no contact in free-space motion, $\mathbf{F}=\mathbf{F}_{\mathbf{r}}=\mathbf{0}$, we obtain:

$$
\mathbf{M}_{\mathbf{d}}\left(\ddot{\mathbf{X}}-\ddot{\mathbf{X}}_{\mathbf{r}}\right)+\mathbf{B}_{\mathbf{d}}\left(\dot{\mathbf{X}}-\dot{\mathbf{X}}_{\mathbf{r}}\right)+\mathbf{K}_{\mathbf{d}}\left(\mathbf{X}-\mathbf{X}_{\mathbf{r}}\right)=\mathbf{0}
$$

Thus, when $\mathbf{X}-\mathbf{X}_{\mathbf{r}} \rightarrow \mathbf{0}$ is achieved, position points will be tracked. The dynamic interaction between the ground and the foot tip will be dictated by (1), if the leg is in supporting phase. In order to simplify, each Cartesian variable is considered independently as (3) in the 


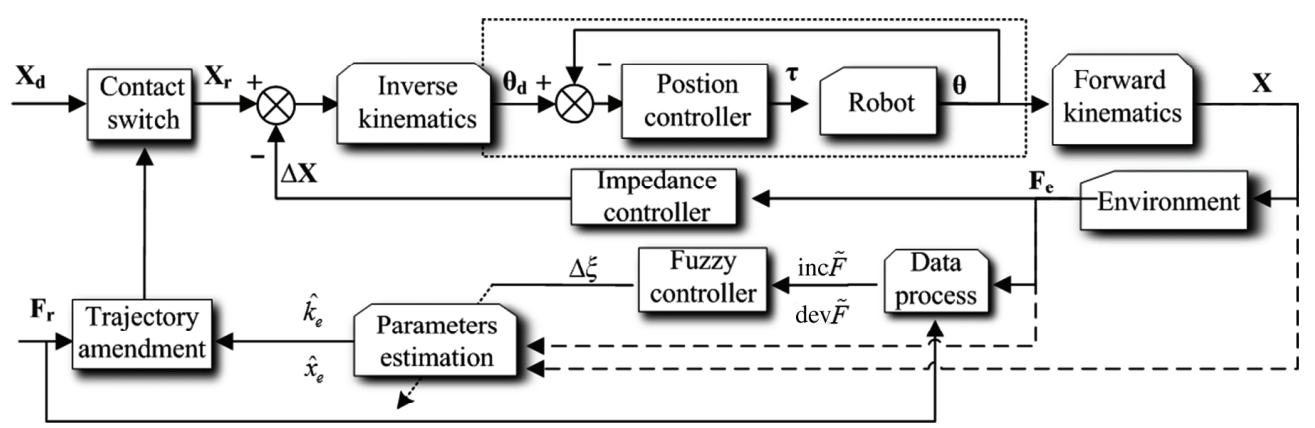

Figure 4. Control scheme of fuzzy-adaptive control.

subsequent analysis. The steady-state contact force can be obtained as (4):

$$
\begin{array}{r}
m_{d}\left(\ddot{x}-\ddot{x}_{r}\right)+b_{d}\left(\dot{x}-\dot{x}_{r}\right)+k_{d}\left(x-x_{r}\right)=-F \\
e_{s s}=F_{r}+\frac{k_{d} k_{e}}{k_{d}+k_{e}}\left(x_{e}-x_{r}\right), F_{s s}=\frac{k_{d} k_{e}}{k_{d}+k_{e}}\left(x_{r}-x_{e}\right)
\end{array}
$$

in which $F_{r}$ is the reference force and is bounded by force planning generator, $x_{e}$ and $k_{e}$ are the position and stiffness of environment, $e$ is the error between $F_{r}$ and $F$. If the reference position trajectory is chosen precisely as (5), then we have $F_{s s} \rightarrow F_{r}, e_{s s} \rightarrow 0$, since the values of $x_{r}$ and $k_{e}$ are not known perfectly. As a result, the desired force $F_{r}$ will not be exerted on environment. Thus, this problem has to be solved. Here, the indirect adaptive method is utilized:

$$
x_{r}=\frac{F_{r}}{k_{e q}}+x_{e}, k_{e q}=\frac{k_{d} k_{e}}{k_{d}+k_{e}}
$$

\section{Improved Adaptive Control Scheme}

\subsection{Indirect Adaptive Control}

The environmental parameters $k_{e q}$ and $x_{e}$ are estimated on-line by an indirect adaptive control scheme [13]. The required reference position $x_{r}$ is calculated, and then these estimates are used to produce the expected force $F_{r}$ in real time.

In former presentation, if $x_{e}$ and $k_{e q}$ has already known, and $x_{r}=\frac{F_{r}}{k_{e q}}+x_{e}$ is specified, then it yields force tracking perfectly with $e_{s s}=0$. Define $x_{r}$ as $x_{r}=\frac{F_{r}}{\hat{k}_{e q}}+\hat{x}_{e}$, where $\hat{x}_{e}$ and $\hat{k}_{e q}$ are denoted as computed estimates of $x_{e}$ and $k_{e q}$, respectively. $k_{e q}=\frac{k_{d} k_{e}}{k_{d}+k_{e}}, \hat{k}_{e q}=\frac{k_{d} \hat{k}_{e}}{k_{d}+\hat{k}_{e}}$.

$k_{d}$ is normally a known constant. The force-tracking method by indirect adaptive can be concluded as (6), which is derived through Lyapunov-based approach:

$$
\begin{cases}\hat{k}_{e}(t)=\hat{k}_{e}(0)-\xi_{1} \int_{0}^{t} x(\hat{F}-F) d t, \quad \hat{x}_{e}(t)=\hat{x}_{e}(0)+\xi_{1} \int_{0}^{t} \frac{(\hat{F}-F)}{\hat{k}_{e}}\left(x \hat{x}_{e}+\xi_{2} / \xi_{1}\right) d t \\ \hat{F}=\hat{k}_{e}\left(x-\hat{x}_{e}\right), \quad x_{r}=\hat{x}_{e}+\frac{F_{r}}{\hat{k}_{e q}}, \quad \hat{k}_{e q}=\frac{\hat{k}_{e} k_{d}}{\hat{k}_{e}+k_{d}}\end{cases}
$$

where $\xi_{1}$ and $\xi_{2}$ are positive constants. The indirect adaptive scheme is derived by using a "certainty equivalence" approach, in which the environmental parameters $x_{e}$ and $k_{e}$ are estimated on-line and the estimates $\hat{x}_{e}$ and $\hat{k}_{e}$ are used to compute $x_{r}$ as if they were equal to the true parameters. It is mentioned that such schemes tend to be robust to unmodelled effects and sensor noise [13].

As is known in (6), the control performance can be influenced significantly by the values of $\xi_{1}$ and $\xi_{2}$, since they are the main coefficients of $\hat{x}_{e}$ and $\hat{k}_{e}$, which determine the performance directly. When $\xi_{1}$ is constant, small $\xi_{2}$ will achieve slow response speed and long rise time, and large $\xi_{2}$ leads to the unacceptable overshoot. As same as $\xi_{2}, \xi_{1}$ must also be chosen properly. Usually, $\xi_{1}$ and $\xi_{2}$ are selected in certain $k_{e}$, so bad performance will happen if the environment changes. Actually, the robot is always walking in an unknown environment, so the stiffness is unknown and is changing on time. When $\xi_{1}, \xi_{2}$ and environment stiffness are too large, the value of $(\hat{F}-F)$ will shock and will not converge. Thus, selection of the parameters value cannot be arbitrary for the stability concern [17]. For the purpose of on-line parameters adjustment, a fuzzy controller is used. The environment stiffness will be changed to raise the response speed and reduce the overshoot.

\subsection{Fuzzy Control Scheme}

The improved compliance control scheme is shown in Fig. 4. There are two varieties of inputs to the fuzzy controller. Contact force deviation $\operatorname{dev} F(k)$ between current force and steady-state force $F_{r}$ is the first one shown in (7). $|\operatorname{sign} F|$ is the contact flag. Since force sensors are usually noisy, a Butterworth low pass filter and a threshold $0.5 \mathrm{~N}$ are used in experiments. The second one is force increment inc $F(k)$ for force derivative as shown in (7). Increments of $\xi_{1}$ which provide direct parameters adjustment are the output of the fuzzy controller. The relationship between $\xi_{1}$ and $\xi_{2}$ 


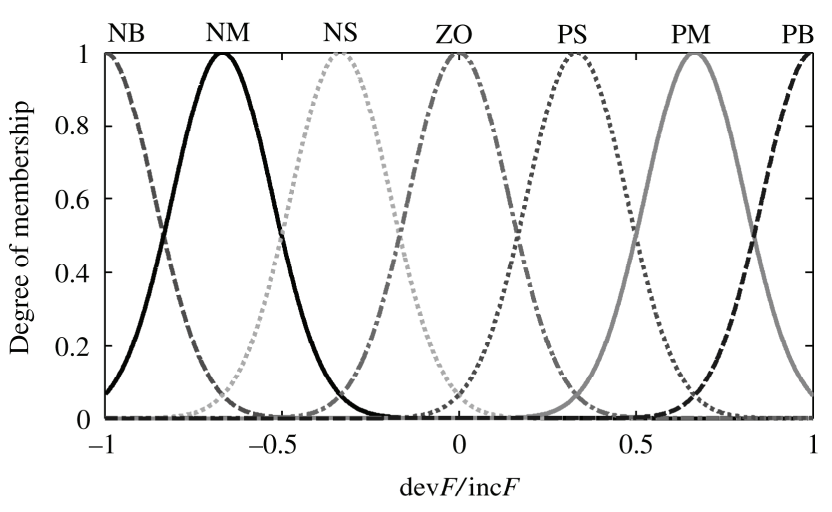

Figure 5. Membership function.

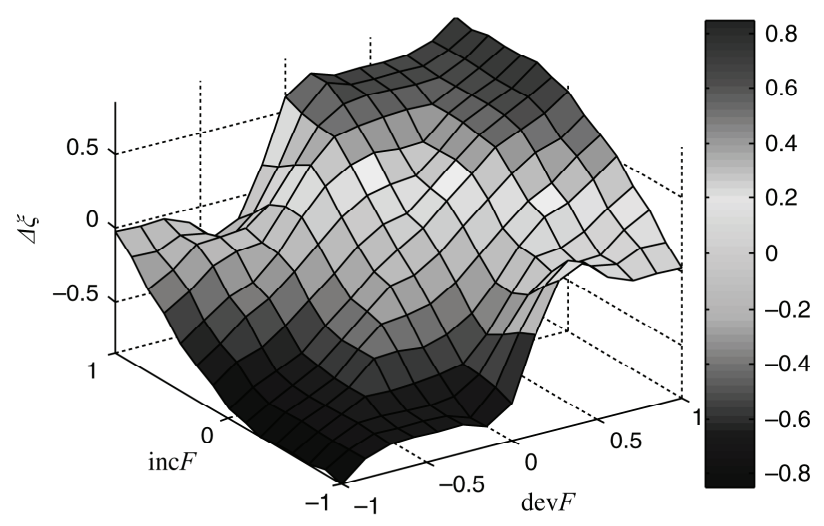

Figure 6. Control surface of the output.

is denoted as (8) for their same effect, in which $\beta$ is the correlation coefficient to decide the importance:

$\operatorname{dev} F(k)=\left(F_{r}-F(k)\right) \cdot|\operatorname{sign} F|, \quad \operatorname{inc} F(k)=F(k)-F(k-1)$

$$
\xi_{2}=\beta \cdot \xi_{1}
$$

Corresponding normalizing gains are used for input variables domains transformation to interval $[-1,1]$. There is only one output $\Delta \xi(k)$. Output of the controller will be transformed to original domain to gain the control decision. Fuzzy partitioning of the input and output controller variables has been initially performed by choosing seven
Table 2

Fuzzy Controller Rules

\begin{tabular}{|c|c|c|c|c|c|c|c|c|}
\hline \multicolumn{2}{|c|}{$\Delta \xi$} & \multicolumn{7}{|c|}{$\operatorname{dev} F$} \\
\cline { 2 - 9 } inc $F$ & NB & NB & NB & NB & NB & NS & NS & ZO \\
\cline { 2 - 9 } & NM & NM & NM & NM & NM & ZO & ZO & ZO \\
\cline { 2 - 9 } & NS & NM & NM & NS & NS & ZO & ZO & NS \\
\cline { 2 - 9 } & ZO & NB & NM & NS & ZO & PS & PM & PB \\
\cline { 2 - 9 } & PS & PS & ZO & ZO & PS & PS & PM & PM \\
\cline { 2 - 9 } & PM & ZO & ZO & ZO & PM & PM & PM & PM \\
\cline { 2 - 9 } & PB & ZO & PS & PS & PB & PB & PB & PB \\
\hline
\end{tabular}

primary fuzzy sets for each variable, which are marked with corresponding linguistic labels that appear in fuzzy control rules, and for which Gaussian membership functions have been selected.

The functions for $\operatorname{dev} F(k), \operatorname{inc} F(k)$ and $\mu$ are shown in Fig. 5. A membership function is a curve that defines how each point in the input space is mapped to a membership value between 0 and 1 . In this case, the Gauss membership functions used for all variables are $\{-1,-0.667$, $-0.333,0,0333,0.667,1\}$ and physical domain is selected for all variables based on trial and error method. The fuzzy variables are defined for the rule base as: $\operatorname{dev} F(k)$, $\operatorname{inc} F(k)=$ the deviation of force, the increment of force, the control variable $\{\mathrm{NB}$ (Negative Big), NM (Negative Medium), NS (Negative Small), ZO (Zero), PS (Positive Small), PM (Positive Medium), PB (Positive Big)\}, $[-1,1], \mu\}$.

The fuzzy rule base is constructed by using several if-then statements, premise and consequence of each statement, which are fuzzy propositions. Table 2 indicates that 49 rules define the rule base for the fuzzy controller. Mamdani's minimum rule is utilized as fuzzy implication function. Centre of gravity (COG) method is used for defuzzification.

The output control surface is shown in Fig. 6, and the adaptive parameters are updated in real time after the interval transformation. The final control scheme can be presented as:

$$
\left\{\begin{array}{l}
\hat{k}_{e}(t)=\hat{k}_{e}(0)-\xi \int_{0}^{t} x(\hat{F}-F) d t, \hat{x}_{e}(t)=\hat{x}_{e}(0)+\xi \int_{0}^{t} \frac{(\hat{F}-F)}{\hat{k}_{e}}\left(x \hat{x}_{e}+\beta\right) d t \\
\hat{F}=\hat{k}_{e}\left(x-\hat{x}_{e}\right), x_{r}=\hat{x}_{e}+\frac{F_{r}}{\hat{k}_{e q}}, \hat{k}_{e q}=\frac{\hat{k}_{e} k_{d}}{\hat{k}_{e}+k_{d}}, \quad \xi(k)=\xi_{0}(k)+\Delta \xi(k)
\end{array}\right.
$$

In this way, the fuzzy controller can regulate the adaptive controller parameters in real time. Simultaneously, the characteristic of the system performance will be adjusted according to the actual encountered situation. Some experiments for comparison are conducted to prove that the proposed method is valid and practical.

\section{Experiments}

For experiments, the stride length is $0.15 \mathrm{~m}$. The body attitude is set $0.2 \mathrm{~m}$. Covering factor is 0.75 and gait cycle is $8 \mathrm{~s}$ in order to highlight the results of compliance control. Experiments in soft ground, hard ground, and 
Table 3

Parameters of Experiments

\begin{tabular}{|c|c|c|c|c|c|}
\hline \multicolumn{2}{|c|}{ Parameters } & Values & \multicolumn{2}{|c|}{ Parameters } & Values \\
\hline Electric resistance & $R_{a}(\Omega)$ & 9.72 & Gear reduction rate & $N_{G}$ & 200 \\
\hline Back EMF constant & $k_{E}(\mathrm{rpm} / \mathrm{V})$ & 563 & Effective inertia & $J_{\text {eff }}\left(\mathrm{gcm}^{2}\right)$ & 2.19 \\
\hline Torque constant & $\begin{array}{c}k_{M} \\
(\mathrm{mNm} / \mathrm{A})\end{array}$ & 17 & Effective damping coefficient & $B_{e f f}(\mathrm{mNm} / \mathrm{rpm})$ & 4.4 \\
\hline \multirow{3}{*}{$\begin{array}{l}\text { Impedance control } \\
\text { parameters }\end{array}$} & $m_{d}$ & 1 & Motor and gear efficiency & $\eta$ & 0.7 \\
\hline & $b_{d}$ & 150 & Correlation coefficient & $\beta$ & 1 \\
\hline & $k_{d}$ & 200 & \multirow{4}{*}{$\begin{array}{l}\text { Indirect adaptive } \\
\text { method parameters }\end{array}$} & $\xi_{1}$ & 80 \\
\hline \multirow{3}{*}{$\begin{array}{l}\text { Fuzzy controller } \\
\text { normalizing gains }\end{array}$} & $\gamma_{\Delta \xi}$ & 5 & & $\xi_{2}$ & 80 \\
\hline & $\gamma_{\operatorname{dev} F}$ & 50 & & $\hat{x}_{e}(0)$ & 2,000 \\
\hline & $\gamma_{\text {inc } F}$ & 0.4 & & $\hat{k}_{e}(0)$ & 10,000 \\
\hline
\end{tabular}

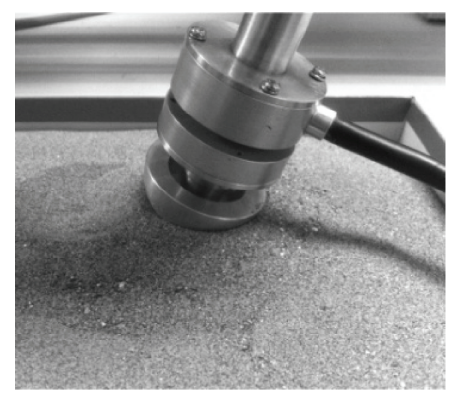

(a)

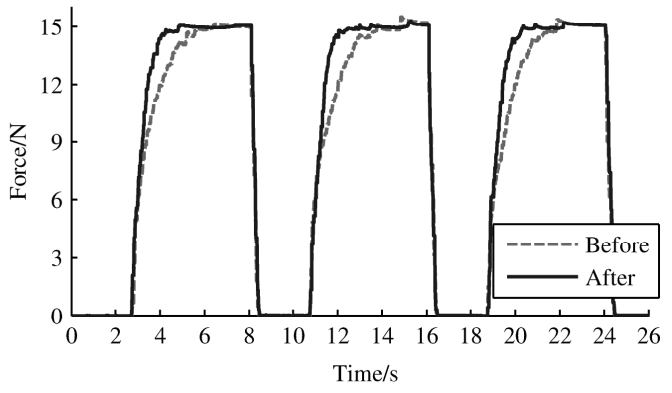

(b)

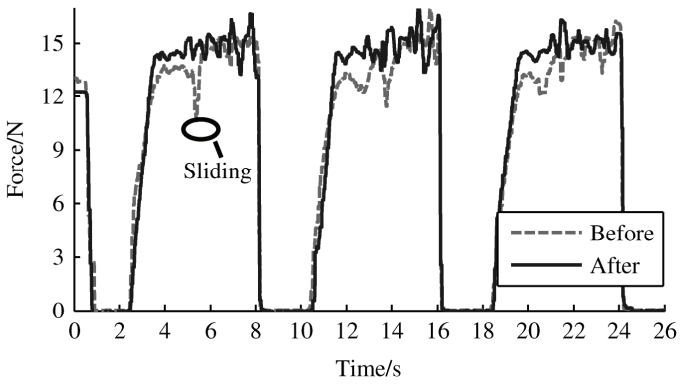

(c)

Figure 7. Walking on sand with desired force $15 \mathrm{~N}$ : (a) photo; (b) static supporting; and (c) walking.

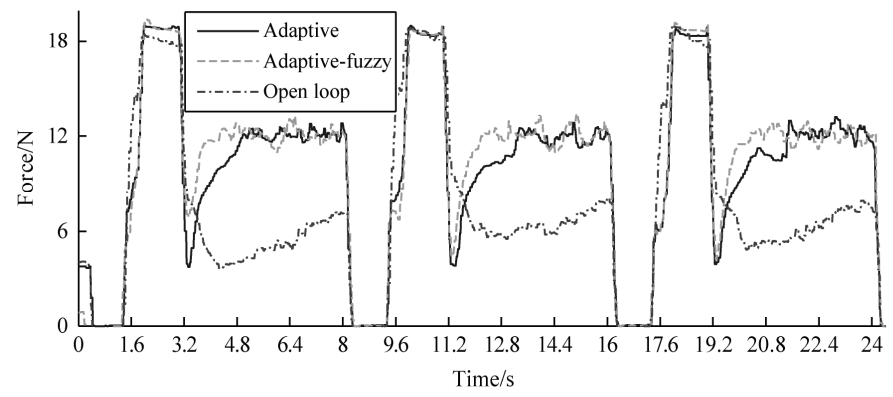

(a)

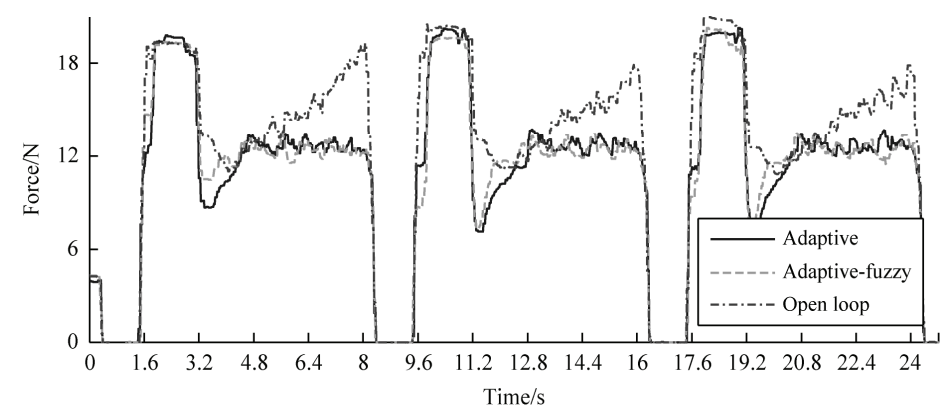

(b)

Figure 8. Foot tip force curves with desired force $12 \mathrm{~N}$ : (a) walking on the sand and (b) walking on hard ground.

sandy ground are conducted. Correlation parameters are shown in Table 3. Both methods use the same parameters.

\subsection{Walking on Sand}

With fuzzy improvement, the proposed method performs excellently in "soft". Figure 7(b) is static supporting force on sand, and there are no contact point changes between ground and foot tip. Steady-state errors are less than $0.4 \%$ in both algorithms and the rate of response is fast. Figure 7(c) is force curve on sand. The curve rises slowly in traditional algorithm and comes about slipping when it reaches $80 \%$ of the expected force for the sand subsidence. However, improved algorithm gets a fast rise during the whole tracking process. The contact forces are fluctuating around the expected force in both algorithms at steady state. This is because the contact point at foot tip is changing all the time. But the range of the fluctuations is acceptable for the whole walking process.

\subsection{Different Circumstances}

Figure 8 includes foot tip force curves of robot walking on sand and hard ground. When a leg is swinging, the other 


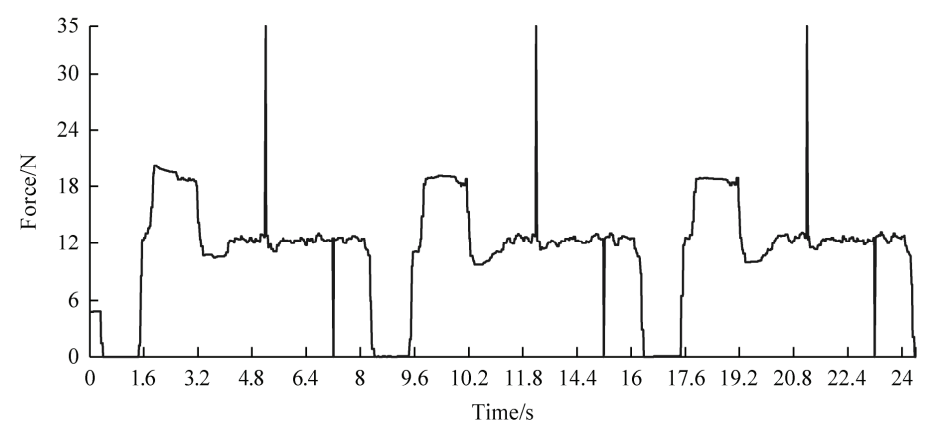

(a)

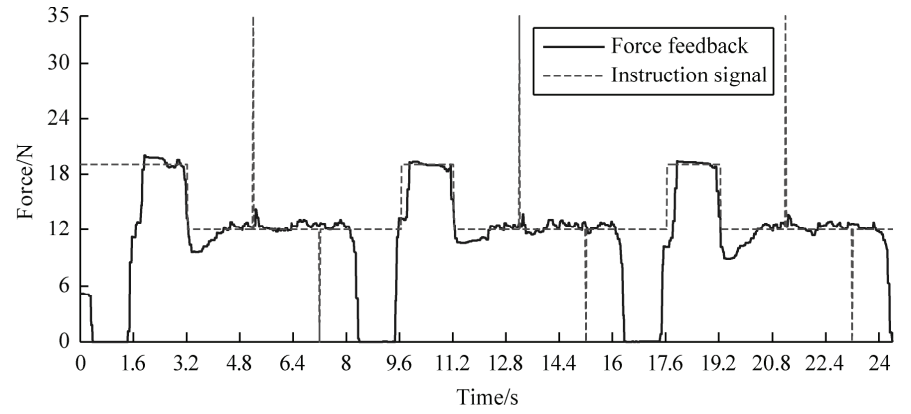

(b)

Figure 9. Foot tip force curves (desired force $=12 \mathrm{~N}$ ) with (a) force sensor disturbs and (b) instruction disturbs.

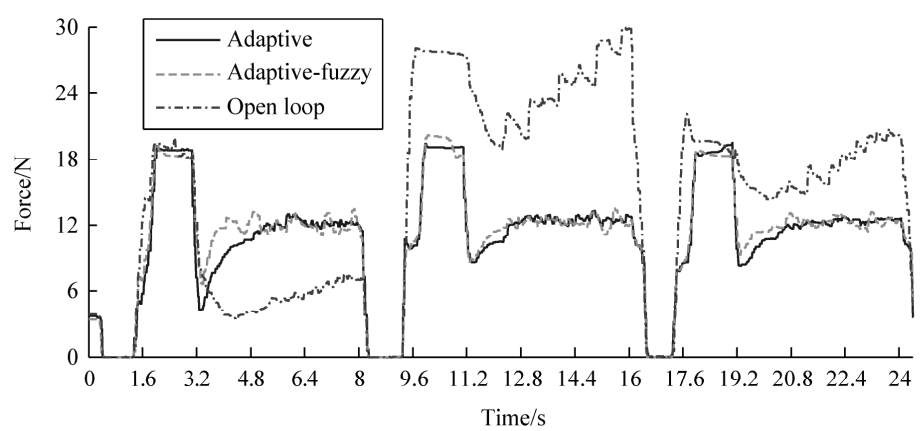

(a)

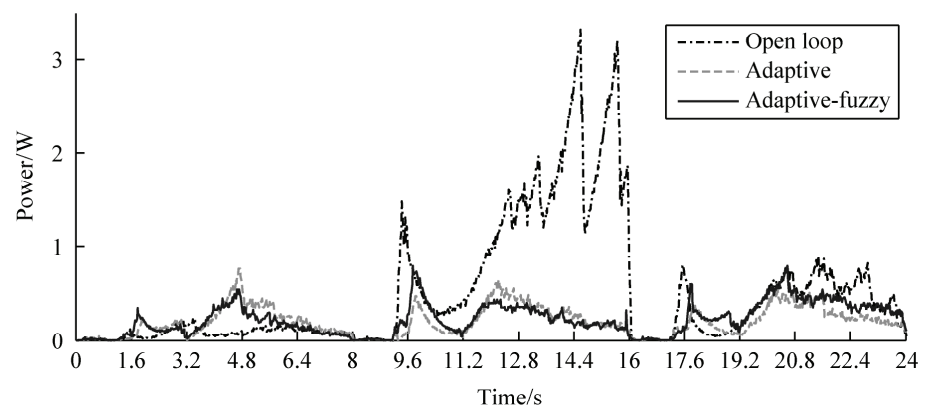

(c)

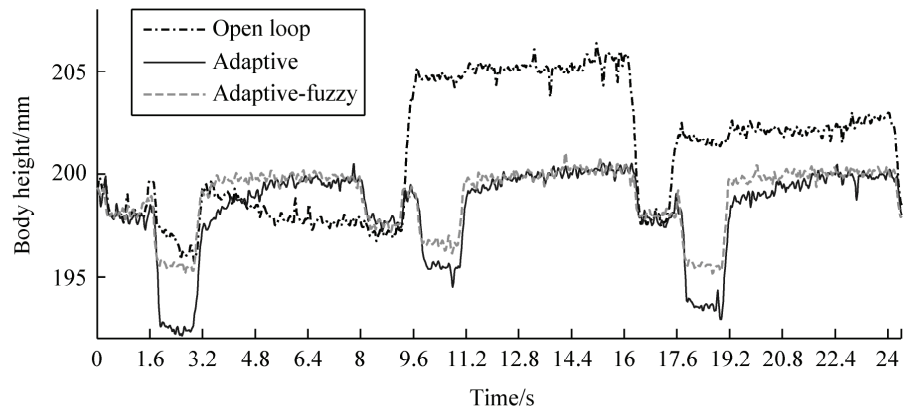

(b)

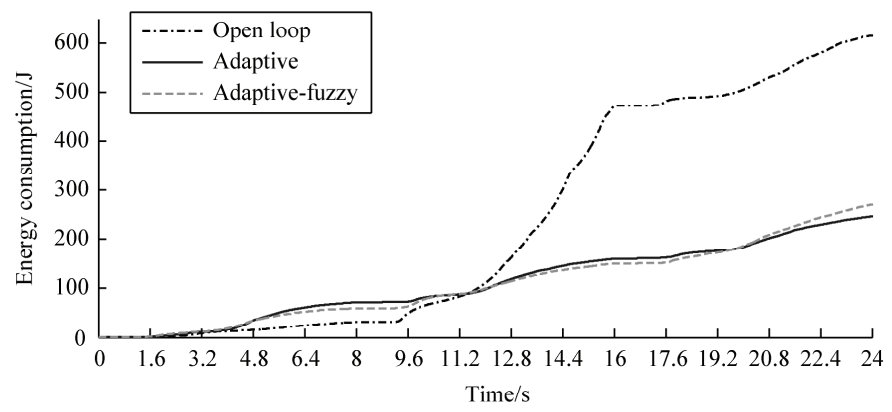

(d)

Figure 10. Walking on different grounds with desired force $12 \mathrm{~N}$ : (a) foot tip force curve; (b) body height variation; (c) power of walking; and (d) system energy consumption.

leg will bear all the weight of body, so the foot force of supporting leg is almost equal to the overall weight of the robot in 1.6-3.2 s. But in 3.2-8 s, both legs are contacted with ground, which contribute to the overall weight of robot.

In Fig. 8(a), the robot foot tip force under openloop control has only soft contact with sand, and the body weight distribution is unreasonable. However, in the adaptive control and adaptive-fuzzy control, despite some impact $(3.2-4 \mathrm{~s})$ exists when the swinging leg is dropping onto the ground, the foot force can be adjusted to achieve the desired force $(12 \mathrm{~N})$ quickly. The raise time is about $0.4 \mathrm{~s}$ in the adaptive-fuzzy control, which has a much faster response than that in the adaptive control. Although the sand ground is soft, the control algorithm makes the robotenvironment system to be "hard" by greatly increasing the target stiffness. In Fig. 8(b), due to the originally larger environment stiffness, the performance enhancement of adaptive-fuzzy control is slight. But in the open-loop control, the test leg carries more weight and the foot tip force changes a lot in walking, since force is mainly influenced by the position control, physical structure, and environment. Thus, the variation trend of foot tip force cannot be predicted and tracked.

\subsection{Under Presence of Disturbs}

Figure 9 shows foot tip force curves under adaptive-fuzzy control with force sensor disturb and instruction disturb, respectively. In Fig. 9(a), disturbs occur in feedback signals of force sensor at values of 35 and $0 \mathrm{~N}$ at the time of 5.8 and $7.4 \mathrm{~s}$, which may be caused by transmission errors. Figure 9(b) shows that disturbs occur in instruction signals at the time of 5.2 and $7.2 \mathrm{~s}$, and the values of disturbs are 
35 and $0 \mathrm{~N}$. The robot only makes minor adjustments to continue approaching to the desired force. The main reason for this phenomenon is that the adaptive-fuzzy control process is actually a process of integral approximation, and the control scheme of the robot movement only made minor adjustments in each control cycle, so the short-term disturbs will not cause too much impact to the robot.

\subsection{Body Height and Energy Consumption}

Compliance control affects the foot tip force of robot most directly. Not only it is limited to the effect on the foot force, but also it demonstrates body height variation and system power consumption. In Fig. 10, foot tip force curve, body height, power, and system energy consumption of robot walking on different grounds are shown. The first cycle is for sand, the second cycle is for hard, and the third cycle is for soft ground. In the process from swinging phase to supporting phase, open-loop control system relies more on foot tip trajectory planning, so it is bound to be influenced seriously by the ground position estimation and environmental stiffness. This can be seen in Fig. 10(a). However, in the compliance control strategies, desired foot force can be quickly tracked and actual foot forces in different environments are all maintained steadily at $12 \mathrm{~N}$. The adaptive-fuzzy control can still show better performance of force tracking than traditional adaptive control in environment with a small stiffness, which can be seen in Fig. 10(a).

In Fig. 10(b), when the foot tip force of test leg is small, the value of body height under open-loop control decreases. But large foot force raises the body rapidly and the value fluctuates about $8 \mathrm{~mm}$. However, in the adaptive control, the fluctuations of body height significantly eliminate, but they still exist at the beginning of landing on sand and soft grounds. In the adaptive-fuzzy control, there is no significant change in body height, although the environment is changing. The body height is always maintained at $0.2 \mathrm{~m}$ with tiny fluctuations. In Fig. 10(c) and (d), it is also very easy to get that the energy consumption in open-loop control is mainly used to do work for the body to influence the body height. Therefore, compliance control can minimize that part of energy consumption which has no benefits to robot, on the premise of stable and smooth walking. That is to say, it can reach purposes of foot force control and energy consumption reduction.

\section{Conclusion}

This article presents an improved compliance control strategy for purpose of impact reduction and stable walking of a hexapod robot under different environments. Adaptive control parameters are adjusted according to different environments. The fuzzy controller makes a faster convergence speed be maintained in whatever environment and foot force regulating speed of the legs can be more balanced by adjusting the parameters, thus stable walking on unknown complex terrain can be guaranteed. Both strategies are analysed through a series of experiments in different circumstances, presence of disturbs, and body height.
The results show that the improved one can achieve a fast response in more complex environments. Although this article only makes a simple authentication on compliance control, it is able to demonstrate the feasibility and effectiveness of the proposed algorithm. The significance of the compliance control on the walking robot is also shown. Hence, this part of the research remains very important for the hexapod robot walking out of laboratory environment independently.

\section{Acknowledgement}

This project is supported by National Natural Science Foundation of China (No. 51605039), Natural Science Basic Research Plan in Shaanxi Province of China (No. 2016JQ6066), Project funded by China Postdoctoral Science Foundation (No. 2016M592728), and Fundamental Research Funds for the Central Universities (No. 310825151041).

\section{References}

[1] Y. Lin and S.M. Song, Learning hybrid position/force control of a quadruped walking machine using a CMAC neural network, Journal of Robotic Systems, 14(6), 1997, 483-499.

[2] C.A. Klein, K.W. Olson, and D.R. Pugh, Use of force and attitude sensors for locomotion of a legged vehicle over irregular terrain, The International Journal of Robotics Research, 2(2), 1983, 3-17.

[3] A. Irawan and K. Nonami, Optimal impedance control based on body inertia for a hydraulically driven hexapod robot walking on uneven and extremely soft terrain, Journal of Field Robotics, 28(5), 2011, 690-713.

[4] M.F. Silva, J.A. Machado, and R.S. Barbosa, Complex-order dynamics in hexapod locomotion, Signal Processing, 86(10), 2006, 2785-2793.

[5] A. Rönnau, T. Kerscher, and R. Dillmann, Dynamic position/ force controller of a four degree-of-freedom robotic leg, Lecture Notes in Control \& Information Sciences, 422, 2012, 117-126.

[6] M. Oku, H. Koseki, H. Ohroku et al., Rough terrain locomotion control of hydraulically actuated hexapod robot COMET-IV, Proceedings of 2008 JSME Conference on Robotics and Mechatronics, Nagano, Japan, 2008.

[7] M. Pavone, P. Arena, L. Fortuna et al., Climbing obstacle in biorobots via $\mathrm{CNN}$ and adaptive attitude control, International Journal of Circuit Theory and Applications, 34(1), 2006, 109-125.

[8] P. Yin, P. Wang, M. Li et al., A novel control strategy for quadruped robot walking over irregular terrain, Proceedings of 2011 IEEE Conference on Robotics, Automation and Mechatronics, Qingdao, China, 2011, 184-189.

[9] J.A. Galvez, J. Estremera, and P. Gonzalez De Santos, A new legged-robot configuration for research in force distribution, Mechatronics, 13(8), 2003, 907-932.

[10] F. Palis, V. Rusin, and A. Schneider, Adaptive impedance/force control of legged robot systems [C], Proceedings of International Conference on Climbing and Walking Robots, Kalsruhe, Germany, 2001, 323-329.

[11] H.J. Liu and K.Y. Young, Applying wave-variable-based sliding mode impedance control for robot teleoperation, International Journal of Robotics 83 Automation, 26(3), 2011, 296-304.

[12] R. Portillo-Vélez, J. de, A. Rodriguez-Angeles, and C.A. CruzVillar, An optimization-based impedance approach for robot force regulation with prescribed force limits [J], Mathematical Problems in Engineering, 2015, 1-13.

[13] H. Seraji and R. Colbaugh, Force tracking in impedance control, The International Journal of Robotics Research, 16(1), 1997, 97-117.

[14] A. Irawan and T.Y. Yin, Optimizing hexapod robot reconfiguration using hexa-quad transformation, International Journal of Robotics \& Automation, 3(2), 2014, 139-150. 
[15] T.A. Guardabrazo, M.A. Jimenez, and P.G.D. Santos, Analysing and solving body misplacement problems in walking robots with round rigid feet, Robotics $\&$ Autonomous Systems, 54(3), 2006, 256-264.

[16] Y.-G. Zhu, B. Jin, W. Li, and S.-T. Li, Optimal design of a hexapod walking robot based on energy consumption and workspace, Transactions of the Canadian Society for Mechanical Engineering, 38(3), 2014, 305-317.

[17] Y.-G. Zhu, B. Jin, and W. Li, Leg compliance control of a hexapod robot based on improved adaptive control in different environments, Journal of Central South University, 22(3), 2015, 904-913.

\section{Biographies}

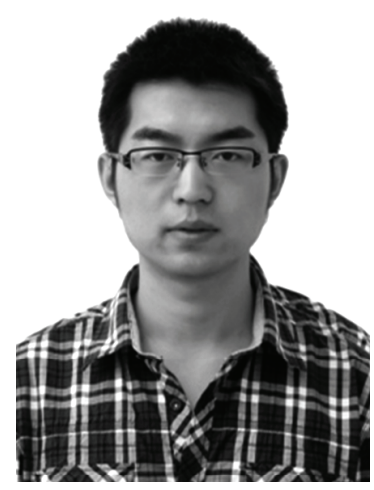

Yaguang Zhu he received the B.S. degree in mechanical and automation engineering from Shandong University, Jinan, China in 2009. He received the Ph.D. degree in mechatronic engineering at Zhejiang University, Hangzhou, China in 2014. He is currently working in Chang'an University. His research interests include robotics, intelligent control, pattern recognition, and electro-

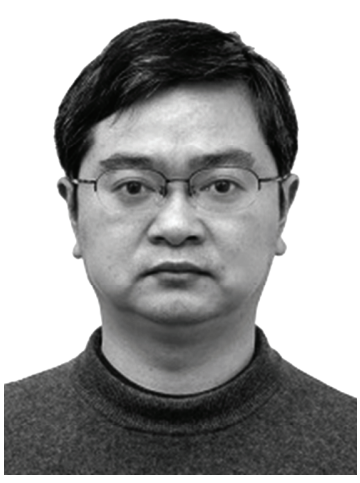

Bo Jin he received the B.S. degree and Ph.D. degree in fluid transmission and control at Zhejiang University, China in 1993 and 1998, respectively. $\mathrm{He}$ is currently a professor at Zhejiang University, attached to the State Key Laboratory of Fluid Power \& Mechatronic Systems. His research interests include deepsea equipment, walking robot, mechatronic control, and electro-

hydraulic control system.

hydraulic control system. 